\title{
THE HASSE INVARIANT OF A VECTOR BUNDLE
}

\author{
BY \\ RICHARD R. PATTERSON
}

\begin{abstract}
The object of this work is to define, by analogy with algebra, the Witt group and the graded Brauer group of a topological space $X$. A homomorphism is defined between them analogous to the generalized Hasse invariant. Upon evaluation, the Witt group is seen to be $\tilde{K} O(X)$, the graded Brauer group $1+H^{1}\left(X ; Z_{2}\right)+$ $H^{2}\left(X ; Z_{2}\right)$ with truncated cup product multiplication, while the homomorphism is given by Stiefel-Whitney classes: $1+w_{1}+w_{2}$.
\end{abstract}

Introduction. The Hasse invariant of a quadratic form on a vector space over a field $k$ is an element of the Brauer group of $k$. It can be generalized to a homomorphism from the Witt group of classes of quadratic forms to the graded Brauer group of classes of graded $k$-algebras [3], [16]. Curiously enough, in this guise it is quite analogous to the homomorphism given by the first two Stiefel-Whitney classes which maps real vector bundles over a space $X$ to the group $1+H^{1}\left(X ; Z_{2}\right)$ $+H^{2}\left(X ; Z_{2}\right)$, where multiplication is by truncated cup product. The purpose of this paper is to display this analogy.

Delzant has defined Stiefel-Whitney classes of quadratic forms [4], [13] using the discriminant and Hasse invariant. It should add insight into his definition to see that the first two topological Stiefel-Whitney classes can also be defined in an algebraic way.

A vector bundle $E$ is known to be orientable for real $K$-theory if its first two Stiefel-Whitney classes vanish. In this case it can be read off from the diagram of Theorem 3.1 that the Clifford bundle of $E$ is a bundle of graded endomorphism algebras of a graded module bundle, which is then used to construct the Thom class of $E$. Donovan and Karoubi in studying this orientability question have done independently much of this same work, applying it further to define $K$-theory with local coefficients [6], [7].

The connection between algebra and topology comes from considering bundles over $X$ whose fibers are vector spaces with quadratic forms or graded algebras and subjecting them to the same analysis the fiber alone is accorded in algebra. This is described in some detail in $\S \mathrm{I}$. §II is devoted to the algebraic computations and §III to a study of the topological problems.

Received by the editors June 23, 1969.

AMS Subject Classifications. Primary 5530, 5730; Secondary 1575, 5732.

Key Words and Phrases. Vector bundles, Witt group, graded Brauer group, Hasse invariant, Clifford bundles.

Copyright (C) 1970, American Mathematical Society 
The author wishes to thank D. R. Anderson for many useful conversations during the course of this work, and the National Science Foundation, grant GP6961, for support.

I. Let $C$ be a category whose objects are real vector spaces, perhaps with additional structure, and whose morphisms are isomorphisms. Let $\perp$ be a binary operation defined on $C$ which is associative and commutative. Then $(C, \perp)$ is called a category with product. A functor between two such is required to preserve the product. A subset of the objects of $C$ is called cofinal if given any object $P$ of $C$ there is another one $Q$ such that $P \perp Q$ is in the subset. The objects of a cofinal subset will be called basic.

We will now give the six examples of categories with product with which we shall be concerned.

1. The category of real vector spaces $R^{n}$ with $n \geqq 0$ will be denoted by $P$. The product is direct sum $\oplus$, and we will consider all objects to be basic.

2. The category of real vector spaces $R^{n}$ with $n>0$ and product the tensor product $\otimes$ will be denoted $F P$. We will again take all objects to be basic.

3. Let $q$ be a nondegenerate quadratic form defined on $R^{n}$ with $n \geqq 0$. We let $Q$ be the category of pairs $\left(R^{n}, q\right)$, with isometries as morphisms and product the orthogonal direct sum. The object $\left(R^{n}, q\right)$ is basic if $q$ has signature zero.

4. We let $A z$ denote the category of Azumaya, or central simple, algebras over the reals. The objects are then $n$-by- $n$ matrix algebras over the reals or the quaternions: $M_{n}(R)$ or $M_{n}(H)$. The morphisms are algebra isomorphisms, the operation is the tensor product, and the $M_{n}(R)$ are basic.

5. We let $F P_{2}$ denote the category of $Z_{2}$-graded vector spaces $R^{p} \oplus R^{q}$ with $p, q \geqq 0, p+q>0$. An element $x$ of $R^{p}$ is called homogeneous of degree $0(d x=0)$, while $y$ of $R^{q}$ is homogeneous with $d y=1$. An isomorphism $f$ of $R^{p} \oplus R^{q}$ is required to be homogeneous of degree zero in the sense that it must send $R^{p}$ to $R^{p}$ and $R^{q}$ to $R^{q}$. The product is the graded tensor product $\hat{\otimes}$; for objects, $\left(R^{p} \oplus R^{q}\right)$ $\hat{\otimes}\left(R^{s} \oplus R^{t}\right)=R^{p s+q t} \oplus R^{q s+p t}$ while for morphisms, $(f \hat{\otimes} g)(x \hat{\otimes} y)=f(x) \hat{\otimes} g(y)$. An object $R^{p} \oplus R^{q}$ is basic if $p=q$.

6. We let $A z_{2}$ denote the category of $Z_{2}$-graded Azumaya algebras. These algebras $A$ are expressible as $A=A^{0} \oplus A^{1}$ with $A^{0}$ nonempty. An element of $A^{0}$ or $A^{1}$ is called homogeneous and we will let $h A$ denote the set of homogeneous elements of $A$. If $a \in h A, d a=i$ for $a \in A^{i}$. We have $d(a b)=d a+d b(\bmod 2)$ for $a, b \in h A$. Morphisms are required to preserve degree. The product is $\hat{\otimes}$, where multiplication in $A \hat{\otimes} B$ is given by $\left(a_{1} \hat{\otimes} b_{1}\right)\left(a_{2} \hat{\otimes} b_{2}\right)=(-1)^{d b_{1} d a_{2} a_{1} a_{2}} \hat{\otimes} b_{1} b_{2}$ for $a_{i} \in h A, b_{i} \in h B$. The product of morphisms is $(f \hat{\otimes} g)(a \hat{\otimes} b)=f(a) \hat{\otimes} g(b)$. Wall [16] has classified these algebras and they are listed in the Table. The basic objects are the graded endomorphism algebras (denoted END $\left(R^{n} \oplus R^{n}\right)$ or $M_{n, n}$ and defined later). This category includes the real Clifford algebras.

Now let $X$ be a finite CW complex. We shall assume that $X$ is connected, as this 
simplifies the discussion and generalizations to nonconnected spaces follow easily. If $C$ is a category with product we define another category $C(X)$ whose objects are isomorphism classes of bundles over $X$. The fiber $P$ of such a bundle is an object of $C$ and the structural group is the automorphism group of $P$ in $C$. Such bundles are in one-to-one correspondence with $[X, B$ Aut $(P)]$, where $B$ Aut $(P)$ is the classifying space of the automorphism group of $P$ [11]. The product in $C$ defines a product in $C(X)$, making $C(X)$ into a semigroup, which we then make into a group $K C(X)$ by the Grothendieck method. In $K C(X)$ the class of a bundle $E$ will be denoted $[E]_{C}$, or just $[E]$ if there will be no confusion. If $X$ is a point this is just the group $K_{0} C$ as defined by Bass [3].

If $f: X \rightarrow Y$ is a continuous map, $f$ induces the usual $f^{*}: K C(Y) \rightarrow K C(X)$. The inclusion of the basepoint into $X$ induces a map rk: $K C(X) \rightarrow K_{0} C$ whose kernel will be denoted $\tilde{K} C(X)$. Thus we get a split short exact sequence

$$
0 \longrightarrow \tilde{K} C(X) \longrightarrow K C(X) \stackrel{\text { rk }}{\longrightarrow} K_{0} C \longrightarrow 0 .
$$

We will now prove that $\widetilde{K} C(X)$ is isomorphic to the group of stable equivalence classes of bundles in $C(X)$.

Call two bundles $E$ and $F$ in $C(X)$ stably equivalent if there exist trivial bundles $P_{1}$ and $P_{2}$ such that $E \perp P_{1}$ and $F \perp P_{2}$ are equivalent. Let $\{E\}_{C}$ (or just $\{E\}$ ) be the stable equivalence class of $E$, and $T C(X)$ the set of classes. Note that we can always find a representative of $\{E\}$ whose fiber is basic. For if the fiber of $E$ is $P$ and $P \perp Q$ is basic, then the product of $E$ and the trivial bundle of fiber $Q$ has a basic fiber and lies in $\{E\}$. We will call a bundle basic if its fiber is basic.

Proposition 1.1. The set $T C(X)$ is a group, which is isomorphic to $\widetilde{K} C(X)$.

Proof. In showing that $T C(X)$ is a group, the only difficulty is the existence of inverses. The proof given by Milnor in [12, Theorem 4.1] for microbundles and adapted by Spivak in [14] for spherical fiber spaces will also work here for our operation $\perp$. The isomorphism from $T C(X)$ to $\widetilde{K} C(X)$ is then given by

$$
\{E\} \rightarrow[E] \perp[\text { rk } E]^{-1} \text {. }
$$

There are product preserving functors defined between these various categories which will unite their study into a single diagram. The hyperbolic functor $H: P \rightarrow Q$ is defined by $H\left(R^{n}\right)=\left(R^{n} \oplus R^{n^{*}}, q\right)$ with $q(x, y)=y(x)$. If $f$ is an isomorphism of $R^{n}$ then $H(f)=f \oplus f^{*-1}$.

Next is End: $F P \rightarrow A z$ which assigns to $R^{n}$ its endomorphism algebra $M_{n}(R)$. An automorphism $\gamma$ of $R^{n}$ goes to inner automorphism by $\gamma$ on $M_{n}(R)$. The analogous graded functor END: $F P_{2} \rightarrow A z_{2}$ assigns to $R^{p} \oplus R^{q}$ its graded endomorphism algebra. The homogeneous elements of

$$
\operatorname{END}^{0}\left(R^{p} \oplus R^{q}\right) \text { or } \quad \operatorname{END}^{1}\left(R^{p} \oplus R^{q}\right)
$$


preserve or reverse degree, respectively. For simplicity of notation we use $M_{p, q}$ for END $\left(R^{p} \oplus R^{q}\right)$. A typical element is a matrix of $M_{p+q}(R)$ with grading indicated by

$$
\left(\begin{array}{ll}
A & B \\
C & D
\end{array}\right)=\left(\begin{array}{ll}
A & 0 \\
0 & D
\end{array}\right)+\left(\begin{array}{ll}
0 & B \\
C & 0
\end{array}\right) .
$$

If $f \in M_{p, q}$ and $g \in h M_{s, t}$ then

$$
(f \hat{\otimes} g)(x \hat{\otimes} y)=(-1)^{d g d x} f(x) \hat{\otimes} g(y) \quad \text { for } x \in h\left(R^{p} \oplus R^{q}\right), \quad y \in R^{s} \oplus R^{t} .
$$

This agrees with the previous definition if $f$ and $g$ are morphisms in the category $F P_{2}$; i.e. isomorphisms in $\mathrm{END}^{0}$. An automorphism of $R^{p} \oplus R^{q}$ is sent by END to inner automorphism of $M_{p, q}$.

The functor $\Lambda: P \rightarrow F P_{2}$ sends $R^{n}$ to $\Lambda^{\text {even }} R^{n} \oplus \Lambda^{\text {odd }} R^{n}$, where $\Lambda^{\text {even }} R^{n}$ is the sum of the even exterior powers of $R^{n}$ and $\Lambda^{\text {odd }} R^{n}$ the odd. A linear transformation of $R^{n}$ extends in the usual way to a morphism of $\Lambda R^{n}$.

And $\mathrm{Cl}: Q \rightarrow A z_{2}$ assigns to $\left(R^{n}, q\right)$ the Clifford algebra it generates [2]. An isometry of $\left(R^{n}, q\right)$ extends to an algebra automorphism of $\mathrm{Cl}\left(R^{n}, q\right)$.

Theorem 1.2. (See Bass [3].) The following diagram is commutative:

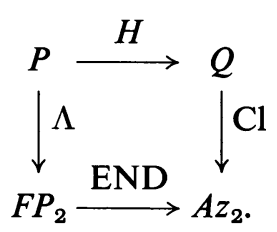

Because these functors preserve products we get immediately a diagram

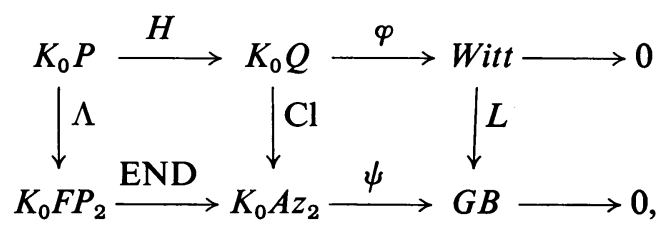

where Witt is the Witt group of the reals, and $G B$ is the graded Brauer group as defined by Wall [16]. The diagram can be regarded as defining Witt and $G B$, with $\varphi$ and $\psi$ the natural projections onto the two cokernels. The induced map $L$ is a generalization of the classical Hasse invariant.

Because these functors naturally extend to bundles we can define two more diagrams.

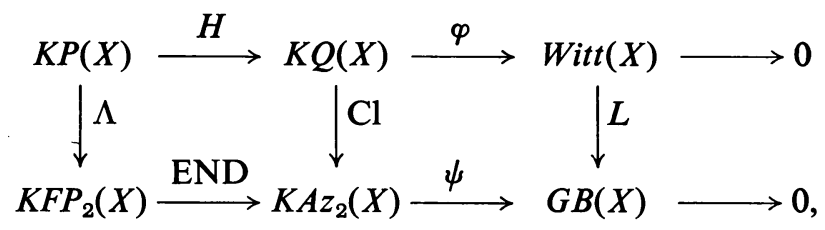

and 
(3)

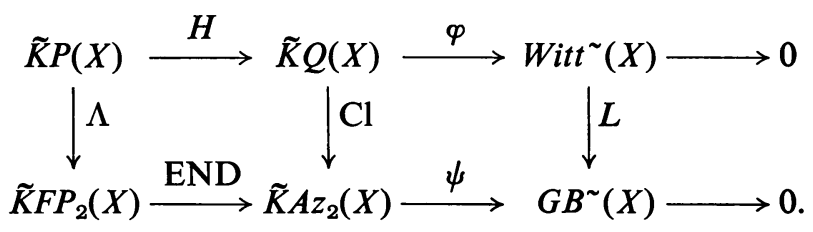

In (2), Witt $(X)$ and $G B(X)$ are defined to be the cokernels of $H$ and END, and similarly in (3). We call Witt ${ }^{\sim}(X)$ the reduced Witt group of $X$ and $G B^{\sim}(X)$ the reduced graded Brauer group. Clearly diagram (2) is known if diagrams (1) and (3) are. It is the comparison of diagrams (1) and (3) which leads to the analogies mentioned in the introduction, and to our definition of $L$ in (3) as a Hasse invariant.

II. This section is devoted to the calculation of the groups and maps of diagram (1).

THEOREM 2.1. The values of the groups and maps in diagram (1) are

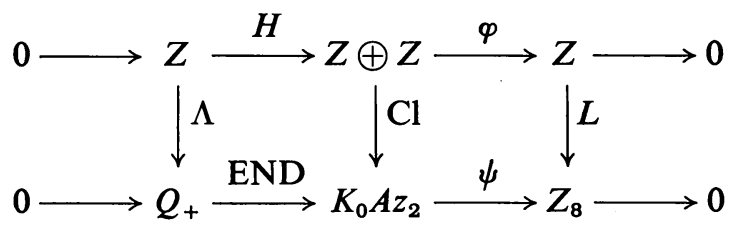

where

(i) The map $H$ is the diagonal and $\varphi(s, t)=s-t$.

(ii) The lower row is a nonsplit extension with $K_{0} A z_{2}$ the index two subgroup of $Q_{+} \sqrt{ } 2 \oplus Z_{8}\left(Q_{+} \sqrt{ } 2\right.$ is the positive rationals with $\sqrt{ } 2$ adjoined $)$ given by all $(a / b, 2 i)$ and $((a / b) \sqrt{ } 2,2 i+1)$. The group operation is multiplication in the first term and addition in the second.

(iii) $\operatorname{END}(a / b)=(a / b, 0)$ and $\psi$ is the projection onto the second term.

(iv) $\Lambda(n)=2^{n}, \mathrm{Cl}(s, t)=\left(2^{(s+t) / 2},(s-t) \bmod 8\right)$, and $L$ is reduction $\bmod 8$.

The proof follows in a sequence of propositions.

We establish the first row as follows. Since the objects of $P$ correspond to the additive semigroup of nonnegative integers, we see that $K_{0} P \cong Z$.

A real nondegenerate quadratic form is given up to equivalence by a diagonal matrix of $s(-1)$ 's and $t(+1)$ 's, thus is completely described by the pair $(s, t)$. Upon including additive inverses we get $K_{0} Q \cong Z \oplus Z$. As $H$ is seen to be the diagonal map, Witt is isomorphic to $Z$, the map $\varphi$ being $\varphi(s, t)=s-t$, the negative of the signature. (A choice made here for later convenience.)

Next we will compute End: $K_{0} F P \rightarrow K_{0} A z$ as an aid for the graded case. The classical Brauer group $B(R)$ is the cokernel of End.

Proposition 2.2. The evaluation of the sequence

$$
K_{0} F P \stackrel{\text { End }}{\longrightarrow} K_{0} A z \longrightarrow B(R) \longrightarrow 0
$$


is $0 \rightarrow Q_{+} \rightarrow Q_{+} \times Z_{2} \rightarrow Z_{2} \rightarrow 0$ which is split by the map $Z_{2} \rightarrow Q_{+} \times Z_{2}$ given by $0 \rightarrow(1,0), 1 \rightarrow(1,1)$.

Proof. The objects of $F P$ comprise the multiplicative semigroup of positive integers, so that $K_{0} F P \cong Q_{+}$, the positive rationals.

The objects of $A z$ can be described as pairs: let $(n, 0)$ correspond to $M_{n}(R)$ and $(2 n, 1)$ to $M_{n}(H)$. The first entry is the square root of the real dimension. If we handle the second entries additively and the first multiplicatively, $A z$ is seen to be the semigroup $Z_{+} \times Z_{2}$ and $K_{0} A z \cong Q_{+} \times Z_{2}$, and $B(R) \cong Z_{2}$, with two elements, $R$ and $H$.

We now begin the graded END sequence.

Proposition 2.3. Let $\alpha: F P \rightarrow F P_{2}$ send $R^{n}$ to $R^{n} \oplus R^{0}$. Then $\alpha$ induces an isomorphism of $K_{0} F P$ and $K_{0} F P_{2}$.

Proof. Define the forgetful functor $\beta: F P_{2} \rightarrow F P$ that sends $R^{p} \oplus R^{q}$ to $R^{p+q}$. Since $R^{p} \oplus R^{q}$ and $R^{s} \oplus R^{t}$ represent the same element of $K_{0} F P_{2}$ if and only if $p+q=s+t$ (tensor each with $R^{1} \oplus R^{1}$ ) we see $R^{p+q} \oplus R^{0}$ and $R^{p} \oplus R^{q}$ are equivalent in $K_{0} F P_{2}$, thus $\alpha$ and $\beta$ are inverse.

The algebras in $A z_{2}$ have been classified by Wall [16] who assigned to each a symbol, given in the Table, column 1 . The corresponding algebra, ignoring the grading, is given in column 2. After placing the algebras in equivalence classes in $G B$, there are eight shortened symbols given in column 3 . These, Wall showed, correspond to mod 8 integers which we indicate in column 4 (actually the inverse to Wall's correspondence), so that $G B$ is isomorphic to $Z_{8}$. The entries in the symbols can best be explained in reference to an exact sequence and the diagram relating the End and END sequences.

The sequence goes as follows. Let $Q(R)$ be the set of 2-dimensional real algebras; i.e. $R \oplus R$ and $C$. Similarly $Q_{2}(R)$ is the set of graded 2-dimensional algebras and there are four of these as each of $R \oplus R$ and $C$ can be graded in two different ways. We can include $Q(R)$ in $Q_{2}(R)$ by concentrating in degree zero, and then using the group operations given by Bass, we have the

Proposition 2.4 [3, Chapter 4, Proposition 3.3]. The sequence

$$
0 \rightarrow Q(R) \rightarrow Q_{2}(R) \rightarrow Z_{2} \rightarrow 0
$$

is exact, and is evaluated

$$
0 \rightarrow Z_{2} \rightarrow Z_{4} \rightarrow Z_{2} \rightarrow 0 \text {. }
$$

For the diagram, we first define $\alpha^{\prime}: A z \rightarrow A z_{2}$ by sending $M_{n}(R)$ to $M_{n, 0}(R)$ and $M_{n}(H)$ to $M_{n, 0}(H)$; that is, to themselves but graded in degree zero. The maps $\alpha$ and $\alpha^{\prime}$ are product preserving, and END $\circ \alpha=\alpha^{\prime} \circ$ End. 
Proposition 2.5. The following diagram is exact and commutative:

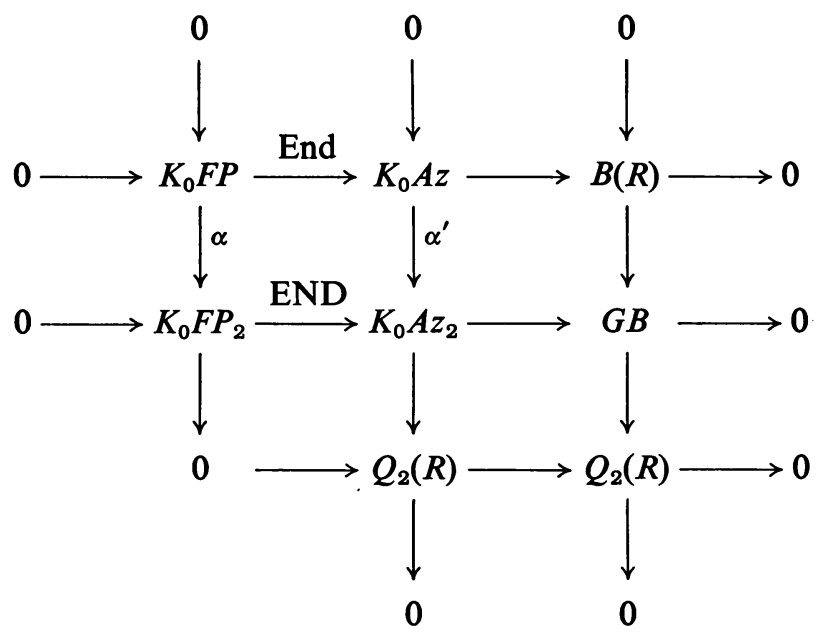

Proof. The right-hand column is proved by Bass [3, Chapter 4, Theorem 4.4]. The maps $\alpha^{\prime}$ and END are monomorphisms, as will be evident after an explanation of the symbols. That coker $\alpha^{\prime} \cong Q_{2}(R)$ follows from the rest of the diagram.

We can now explain the symbols. The $R$ or $H$ refers to an element of $B(R)$, the + or - to the right $Z_{2}$ in Proposition 2.4, the +1 or -1 to $Q(R)$. Then $n$ refers to the size of the matrices as in the Table, and the $(p, q)$ in cases 0 and 4 to the grading as $\operatorname{END}\left(R^{p} \oplus R^{q}\right)$ or $\operatorname{END}\left(H^{p} \oplus H^{q}\right)$. This last bit of information is lost in $K_{0} A z_{2}$ for the same reason it was in $K_{0} F P_{2}$ (Proposition 2.3), so we can ignore it. Building from the symbols to $K_{0} A z_{2}$ is then seen to involve three group extensions, two of which are already done.

Proof of Theorem 2.1. Column 5 of the Table gives a pair which consists of the square root of the real dimension of the algebra and its class in $G B$, which makes

\begin{tabular}{l|c|c|c|l}
\hline Wall's symbols & $\begin{array}{c}\text { Ungraded } \\
\text { algebras }\end{array}$ & $\begin{array}{c}\text { Shortened } \\
\text { symbols }\end{array}$ & $Z_{8}$ & \\
\hline$(+, R,+1, n,(p, q))$ & $M_{n}(R)$ & $(+, R,+1)$ & 0 & $(n, 0)$ \\
$(-, R,+1, n)$ & $M_{n}(R) \oplus M_{n}(R)$ & $(-, R,+1)$ & 7 & $(n \sqrt{ } 2,7)$ \\
$(+, R,-1,2 n)$ & $M_{2 n}(R)$ & $(+, R,-1)$ & 6 & $(2 n, 6)$ \\
$(-, H,-1, n)$ & $M_{2 n}(C)$ & $(-, H,-1)$ & 5 & $(2 n \sqrt{ } 2,5)$ \\
\hline$(+, H,+1, n,(p, q))$ & $M_{n}(H)$ & $(+, H,+1)$ & 4 & $(2 n, 4)$ \\
$(-, H,+1, n)$ & $M_{n}(H) \oplus M_{n}(H)$ & $(-, H,+1)$ & 3 & $(2 n \sqrt{ } 2,3)$ \\
$(+, H,-1, n)$ & $M_{n}(H)$ & $(+, H,-1)$ & 2 & $(2 n, 2)$ \\
$(-, R,-1, n)$ & $M_{n}(C)$ & $(-, R,-1)$ & 1 & $(n \sqrt{ } 2,1)$ \\
\hline
\end{tabular}


clear the semigroup structure in $A z_{2}$ as multiplication in the first factor and mod 8 addition in the second. When we include inverses, we get the group stated in the theorem. The maps are deduced easily from the definitions, except perhaps $\mathrm{Cl}$. The dimension of $\mathrm{Cl}(s, t)$ is $2^{s+t}$ while its graded Brauer class comes from an inspection of the algebras as described in [2]. One can note also that in this notation $\alpha^{\prime}(a / b, i)=(a / b, 4 i)$.

III. In this section we investigate the reduced cohomology theories $\widetilde{K} C$ to find their classifying spaces and evaluate diagram (3).

THEOREM 3.1. The values of the groups and maps in diagram (3) are

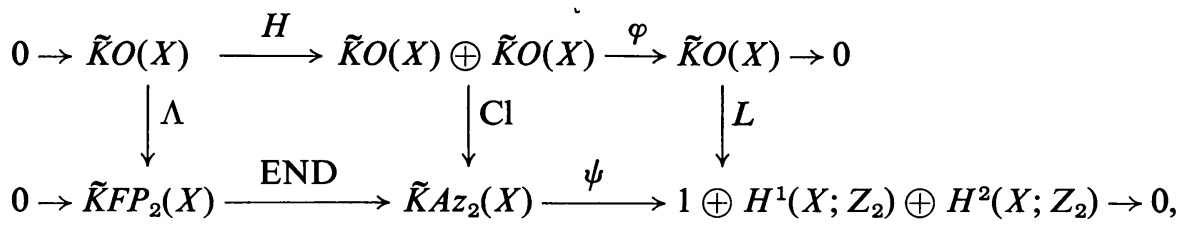

where:

(i) The map $H$ is the diagonal and $\varphi(\{E\},\{F\})=\{E\}-\{F\}$.

(ii) There is an isomorphism between $\tilde{K} F P_{2}(X)$ and $Q \otimes \tilde{K} O(X)$.

(iii) $\psi \circ \mathrm{Cl}(\{E\},\{F\})=\left(1+w_{1}(E)+w_{2}(E)\right)\left(1+w_{1}(F)+w_{1}(F)^{2}+w_{2}(F)\right)$, so that $L(\{E\})=1+w_{1}(E)+w_{2}(E)$.

Again we begin with the first row. Clearly $\tilde{K} P(X)$ is $\tilde{K} O(X)$.

A basic bundle in $Q(X)$ has group the isometry group for some $n$ of the quadratic form $-x_{1}^{2}-x_{2}^{2}-\cdots-x_{n}^{2}+y_{1}^{2}+\cdots+y_{n}^{2}$. Since this group contains $O(n) \times O(n)$ as a deformation retract $[9$, p. 345], every bundle splits into a sum $E \oplus F$, where $E$ has a negative definite and $F$ a positive definite metric. So we can define $\operatorname{Witt}^{\sim}(X)$ and $\varphi$ as indicated.

As before we shall first study End: $\tilde{K} F P(X) \rightarrow \widetilde{K} A z(X)$ as an aid for the graded case. We shall call its cokernel $B^{\sim}(X)$ the reduced (ungraded) Brauer group of $X$.

Proposition 3.2. The classifying space for $F P$ is $B O_{\otimes}$, where $O_{\otimes}$ is the direct limit of the orthogonal groups $O(n)$ under the mappings $\otimes I_{m}: O(n) \rightarrow O(n m)$. There is a commutative diagram of isomorphisms

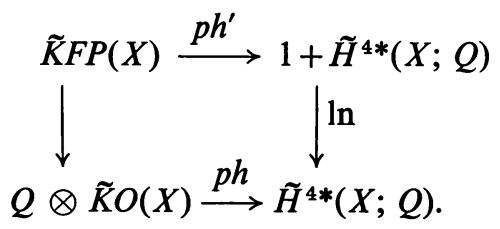

Proof. The first statement is clear, since if $E$ is an $n$-plane bundle and $\varepsilon$ the trivial line bundle, the group of $E \otimes m \varepsilon$ is the image of $O(n)$ in $O(\mathrm{~nm})$. In matrix notation, if $e_{1}, \ldots, e_{n}$ is a basis for $R^{n}$ and $d_{1}, \ldots, d_{m}$ a basis for $R^{m}$, and if we arrange the basis for $R^{n} \otimes R^{m}$ in reverse lexicographical order $e_{1} \otimes d_{1}, e_{2} \otimes d_{1}$, 
$\ldots, e_{n} \otimes d_{m}$, the matrix of $A \otimes I_{m}$ for $A \in O(n)$ is given by $A m$ times down the diagonal.

Define an isomorphism $\delta: \widetilde{K} F P(X) \rightarrow 1+Q \otimes \widetilde{K} O(X)\left[3\right.$, p. 40] by $\delta\{E\}_{F P}$ $=1+1 / \mathrm{rk} E \otimes\{E\}_{P}$. This map is well defined and preserves multiplication. It is surjective, since given any element of $Q \otimes \tilde{K} O(X)$, it can first be written in the form $1 / n \otimes\{E\}_{P}$ with $n>0$. Then choose $r$ such that $r n>\operatorname{dim} X$ and rewrite it as

$$
1 / r n \otimes\{E \otimes r \varepsilon\}_{P}=1 / \mathrm{rk} F \otimes\{F\}_{P}
$$

for that unique bundle $F$ of rank $r n$ in this stable equivalence class. Then $\delta\{F\}_{F P}$ $=1+1 / n \otimes\{E\}_{P}$. And $\delta$ is injective, since if $1+1 / \mathrm{rk} E \otimes\{E\}_{P}=1+1 / \mathrm{rk} F \otimes\{F\}_{P}$, taking $E$ and $F$ of the same dimension $n>\operatorname{dim} X$ we have $1 / n \otimes\left(\{E\}_{P}-\{F\}_{P}\right)=0$, hence $m\{E\}_{P}=m\{F\}_{P}$ for some $m$. Then $E \otimes m \varepsilon=F \otimes m \varepsilon$ or $\{E\}_{F P}=\{F\}_{F P}$.

This map $\delta$ followed by $\ln$ (given by the series expansion of $\ln (1+x)$ ) gives the isomorphism on the left, while $\ln$ itself is used on the right.

The Pontrjagin character ph: $Q \otimes \tilde{K} O(X) \rightarrow \sum_{\imath \geqq 1} H^{4 i}(X ; Q)$ is an isomorphism [10] and because it is multiplicative we can define $\mathrm{ph}^{\prime}\{E\}_{F P}=\mathrm{ph} E / \mathrm{rk} E$. The diagram then commutes, for

$$
\begin{aligned}
\mathrm{ph} \ln \delta\{E\}_{F P} & =\ln \left(1+\mathrm{ph}\left(\frac{1}{\mathrm{rk} E} \otimes\{E\}_{P}\right)\right)=\ln \left(1+\frac{\mathrm{ph}\{E\}}{\mathrm{rk} E}\right) \\
& =\ln \left(1+\frac{\mathrm{ph} E-\mathrm{rk} E}{\mathrm{rk} E}\right)=\ln ^{\mathrm{ph}^{\prime}\{E\}_{F P} .}
\end{aligned}
$$

This completes the proof.

A basic bundle in $A z(X)$ has fiber $M_{n}(R)$. Since by the Skolem-Noether theorem all automorphisms of $M_{n}(R)$ are inner, the structural group of the bundle is the projective orthogonal group $P O(n)$, which is the quotient of $O(n)$ by its center $Z_{2}$. Furthermore if $E$ is a vector bundle with fiber $R^{n}$ then End $E$ has fiber $M_{n}(R)$ and the same coordinate transformations acting by inner automorphism. Thus if $E$ is classified by $f: X \rightarrow B O(n)$, we see that End $E$ is classified by $f$ followed by the natural projection into $B P O(n)$.

Proposition 3.3. The classifying space for $A z$ is $B P O$, where $P O$ is the limit of the projective orthogonal groups under $\otimes I_{m}: P O(n) \rightarrow P O(n m)$. The projection $O(n) \rightarrow P O(n)$ induces End: $\widetilde{K} F P(X) \rightarrow \widetilde{K} A z(X)$ whose cokernel $B^{\sim}(X) \cong H^{2}\left(X ; Z_{2}\right)$. Thus the following sequence is exact:

$$
0 \longrightarrow \tilde{K} F P(X) \stackrel{\text { End }}{\longrightarrow} \tilde{K} A z(X) \longrightarrow H^{2}\left(X ; Z_{2}\right) \longrightarrow 0 .
$$

Proof. The diagram

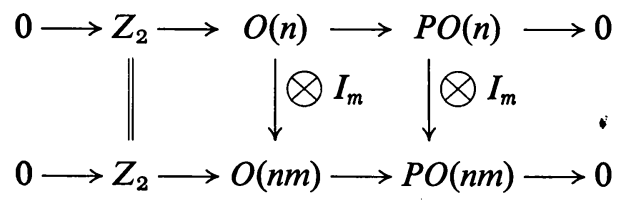


is exact and commutative, hence in the limit there is a short exact sequence $0 \rightarrow Z_{2} \rightarrow O_{\otimes} \rightarrow P O \rightarrow 0$. This gives a sequence of fiber spaces $Z_{2} \rightarrow O_{\otimes} \rightarrow P O$ $\rightarrow K\left(Z_{2}, 1\right) \rightarrow B O_{\otimes} \rightarrow B P O \rightarrow K\left(Z_{2}, 2\right)$. As $Z_{2} \rightarrow O(n)$ for $n$ even is homotopic to the constant map, it follows that $K\left(Z_{2}, 1\right) \rightarrow B O_{\otimes}$ is likewise, so that End is a monomorphism. The Bott Periodicity Theorem and our direct limit system give the homotopy of $B O_{\otimes}$ as $Q$ in every dimension $4 i, i>0$. Hence there can be no obstruction to a cross-section of $B P O \rightarrow K\left(Z_{2}, 2\right)$ and we see that

$$
\tilde{K} A z(X) \rightarrow H^{2}\left(X ; Z_{2}\right)
$$

is onto.

Remark. For the complex numbers, $B^{\sim}(X)$ is the torsion subgroup of $H^{3}(X ; Z)$. See [8].

Added in proof. A proof that $\tilde{K} A z(X) \rightarrow H^{2}\left(X ; Z_{2}\right)$ is onto which avoids the use of the Bott Periodicity Theorem is given in [6]. They show that End is naturally split by the map $\frac{1}{2} j: \widetilde{K} A z(X) \rightarrow \widetilde{K} F P(X)$, where $j$ is induced by the map of $P O(n)$ to $O\left(n^{2}\right)$ sending $\pm \alpha$ to $\alpha \otimes \alpha$. Lemma 3.11 shows that for spheres, $\widetilde{K} A z$ and $\widetilde{K} F P \oplus H^{2}$ agree, and hence by 7.1 of [5], they agree for all finite complexes.

We now begin the graded END sequence.

Proposition 3.4. $\tilde{K} F P_{2}(X) \cong \tilde{K} F P(X)$.

Proof. As with $X$ a point (Proposition 2.3), take $\alpha: F P(X) \rightarrow F P_{2}(X)$ given by $\alpha(E)=E \oplus 0$ and $\beta: F P_{2}(X) \rightarrow F P(X)$ the forgetful functor. The proof is the same.

Before we can properly discuss stabilizing the bundles in $A z_{2}(X)$ we need a matrix formula for the graded tensor product of $M_{n, m}$ and $M_{p, q}$. If the basis for $R^{n} \oplus R^{m}$ is $e_{1}, \ldots, e_{n}, e_{1}^{\prime}, \ldots, e_{m}^{\prime}$, and for $R^{p} \oplus R^{q}$ is $d_{1}, \ldots, d_{p}, d_{1}^{\prime}, \ldots, d_{q}^{\prime}$, we must choose the basis order for $R^{n p+m q} \oplus R^{m p+n q}$. It comes naturally in four subsets, each with the reverse lexicographical ordering:

$$
\begin{aligned}
& e_{1} \otimes d_{1}, e_{2} \otimes d_{1}, \ldots, e_{n} \otimes d_{p} \\
& e_{1}^{\prime} \otimes d_{1}^{\prime}, \ldots, e_{m}^{\prime} \otimes d_{q}^{\prime} \\
& e_{1}^{\prime} \otimes d_{1}, \ldots, e_{m}^{\prime} \otimes d_{p} \\
& e_{1} \otimes d_{1}^{\prime}, \ldots, e_{n} \otimes d_{q}^{\prime} .
\end{aligned}
$$

LEMMA 3.5. With this choice of basis order we have the following formulas for the graded tensor product of two elements of $M_{1,1}$ and for an element of $M_{n, m}$ and the identity matrix $I_{p, q}$.

$$
\left(\begin{array}{ll}
a & b \\
c & d
\end{array}\right) \hat{\otimes}\left(\begin{array}{ll}
e & f \\
g & h
\end{array}\right)=\left(\begin{array}{rr|rr}
a e & -b f & b e & a f \\
c g & d h & -d g & c h \\
\hline c e & -d f & d e & c f \\
a g & b h & -b g & a h
\end{array}\right),
$$




$$
\left(\begin{array}{ll}
A & B \\
C & D
\end{array}\right) \hat{\otimes}\left(\begin{array}{ll}
I & 0 \\
0 & I
\end{array}\right)=\left(\begin{array}{cc|cc}
A \otimes I_{p} & 0 & B \otimes I_{p} & 0 \\
0 & D \otimes I_{q} & 0 & C \otimes I_{q} \\
\hline C \otimes I_{p} & 0 & D \otimes I_{p} & 0 \\
0 & B \otimes I_{q} & 0 & A \otimes I_{q}
\end{array}\right) .
$$

Proof. Evaluate these linear transformations on the basis elements.

REMARK. Unfortunately, due to the choice of ordering of the basis elements, this product is not associative. It would be associative if we did not separate the basis elements into subsets, but this would make the grading confusing.

Define the graded orthogonal group $G O(n)$ to be all matrices in $O(2 n)$ of the form

$$
\left(\begin{array}{ll}
A & 0 \\
0 & A
\end{array}\right) \text { or }\left(\begin{array}{ll}
0 & B \\
B & 0
\end{array}\right)
$$

with $A, B \in O(n)$. Define the projective graded orthogonal group $P G O(n)$ to be $G O(n)$ modulo $\pm I_{n, n}$.

Proposition 3.6. The classifying space for $\mathrm{Az}_{2}$ is $\mathrm{BPGO}$, the limit of the projective graded orthogonal groups under $\hat{\otimes} I_{p, q}: P G O(n) \rightarrow P G O(n(p+q))$.

Proof. A basic bundle in $A z_{2}(X)$ has fiber $M_{n, n}$. We need the structural group. Automorphisms of $M_{n, n}$ are all inner, so we must find the units which preserve the grading. In $M_{n, n}$ are two central idempotents,

$$
u_{1}=\left(\begin{array}{ll}
I & 0 \\
0 & 0
\end{array}\right) \text { and } u_{2}=\left(\begin{array}{ll}
0 & 0 \\
0 & I
\end{array}\right),
$$

which must be preserved or reversed by any inner automorphism. If $\left(\begin{array}{c}A \\ C\end{array}{ }_{D}^{B}\right)$ sends $u_{1}$ to itself, then $B=C=0$. If it sends $u_{1}$ to $u_{2}$, then $A=D=0$. Hence the homogeneous units, modulo their center $R^{*}$, give all the inner automorphisms of $M_{n, n}$.

We wish to argue that stably we may take the structural group to be $P G O(2 n)$. First for reasons of homotopy we can assume that we have only the units $\left(\begin{array}{ll}A & 0 \\ 0 & D\end{array}\right)$ and $\left(\begin{array}{cc}0 & B \\ C & 0\end{array}\right)$ with $A, B, C, D \in O(n)$. We stabilize the bundle once by tensoring with END $(\varepsilon \oplus \varepsilon)$. The lemma then says our coordinate transformations $g_{i j}(x)$ have matrices of the form

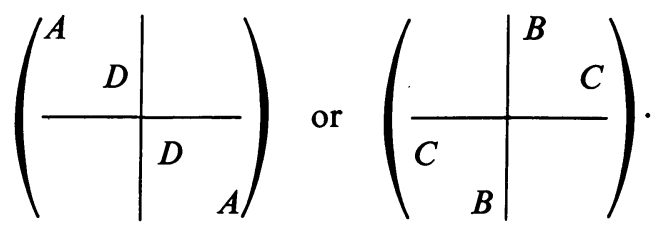

Let

$$
J=\left(\begin{array}{l|ll}
I_{2 n} & & \\
\hline & & I_{n}
\end{array}\right)
$$


The bundle with coordinate transformations $g_{i j}^{\prime}(x)=J g_{i j}(x) J$ is equivalent to our bundle $[15,2.10]$ and here the $g_{i j}^{\prime}(x)$ have matrices of the form

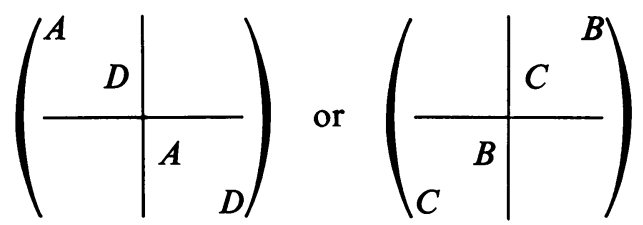

and so are in $G O(2 n)$.

For $G O(n)$ the matrix formulas for $\hat{\otimes}$ reduce to

$$
\left(\begin{array}{l|l}
A & \\
\hline & A
\end{array}\right) \hat{\otimes} I_{p, q}=\left(\begin{array}{l|l}
A \otimes I_{p+q} & \\
\hline & A \otimes I_{p+q}
\end{array}\right)
$$

and

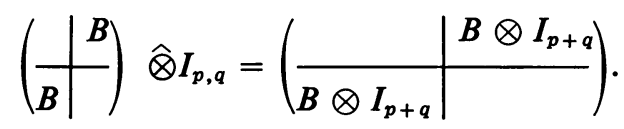

Clearly $\hat{\otimes} I_{p, q}$ and $\hat{\otimes} I_{p^{\prime}, q^{\prime}}$ give the same map if $p+q=p^{\prime}+q^{\prime}$ and if $\hat{\otimes} I_{p, q}$ is followed by $\hat{\otimes} I_{r, s}$, the result is the same as $\hat{\otimes} I_{p r+q s, q r+p s}$. This completes the proof.

The following commutative diagram of exact sequences of groups is necessary for the derivation of the END sequence. Let $\Delta: O(n) \rightarrow G O(n)$ send $A$ to

$$
\left(\begin{array}{l|l}
A & \\
\hline & A
\end{array}\right)
$$

and let $\Delta^{\prime}$ be the induced map, $\Delta^{\prime}: P O(n) \rightarrow P G O(n)$.

(4)

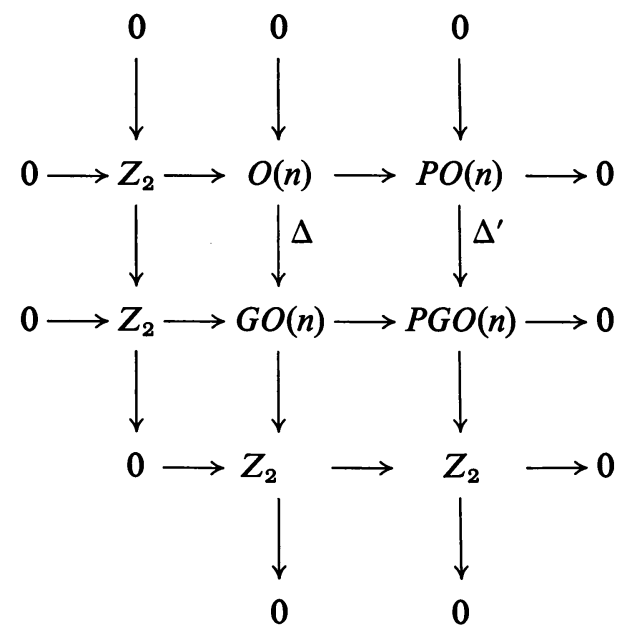


Since these maps commute with our direct limit system, we get in the limit the diagram:

(5)

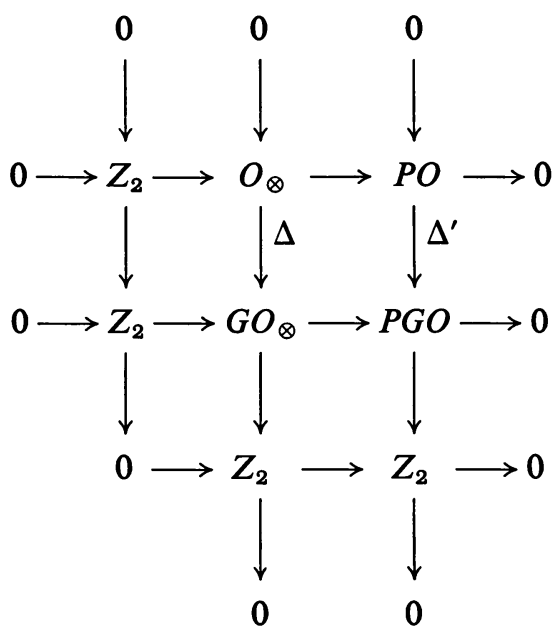

The first row of (5) we have already studied in connection with End. The righthand column is next. Recall the map $\alpha^{\prime}: A z \rightarrow A z_{2}$ which sends $M_{n}(R)$ to $M_{n, 0}$. It defines a map of bundles, $\alpha^{\prime}: A z(X) \rightarrow A z_{2}(X)$.

Proposition 3.7. The map $\alpha^{\prime}: A z(X) \rightarrow A z_{2}(X)$ is induced by $\Delta^{\prime}: P O \rightarrow P G O$ and the following sequence is exact:

$$
0 \longrightarrow \tilde{K} A z(X) \stackrel{\alpha^{\prime}}{\longrightarrow} \tilde{K} A z_{2}(X) \stackrel{T_{*}}{\longrightarrow} H^{1}\left(X ; Z_{2}\right) \longrightarrow 0 .
$$

Proof. A bundle $E$ in $A z(X)$ with fiber $M_{n}(R)$ is to be thought of as having fiber $M_{n, 0}$. Then we form $E \hat{\otimes} \operatorname{END}(\varepsilon \oplus \varepsilon)$. If a coordinate transformation of $E$ is $g_{i j}(x)=A \in P O(n)$, the new bundle has in its place

$$
\left(\begin{array}{l|l}
A & \\
&
\end{array}\right)=\Delta^{\prime}(A)
$$

in $P G O(n)$.

The right-hand column of diagram (5) gives us the following sequence of fibrations:

$$
Z_{2} \longrightarrow B P O \stackrel{\Delta_{*}^{\prime}}{\longrightarrow} B P G O \stackrel{T}{\longrightarrow} K\left(Z_{2}, 1\right),
$$

which gives the exactness of the sequence of the proposition except at the ends. Because $B P O$ is connected, $Z_{2} \rightarrow B P O$ is homotopic to zero, thus $\alpha^{\prime}$ is a monomorphism. The map of $G O(n)$ to $Z_{2}$ in (4) is split by sending 0 to

$$
\left(\begin{array}{l|l}
I & \\
\hline & I
\end{array}\right)
$$


and 1 to

$$
\left(\begin{array}{l|l} 
& I \\
\hline I &
\end{array}\right)
$$

This induces a cross-section to $T$, implying that $T_{*}$ is onto.

REMARK 3.8. If $E$ is a graded algebra bundle, we can think of $T_{*}\{E\}$ as a line bundle with coordinate transformation +1 or $-1 \in O(2)$ where $E$ has coordinate transformation

$$
\left(\begin{array}{l|l}
A & \\
\hline & A
\end{array}\right) \text { or }\left(\begin{array}{l|l}
B \\
\hline B
\end{array}\right) \text {, }
$$

respectively.

Proposition 3.9. There is a one-to-one correspondence between $G B^{\sim}(X)$ and $H^{1}\left(X ; Z_{2}\right) \times H^{2}\left(X ; Z_{2}\right)$. Thus the following sequence is exact: (as sets at $\left.H^{1} \times H^{2}\right)$

$$
0 \longrightarrow \tilde{K} F P_{2}(X) \stackrel{\text { END }}{\longrightarrow} \tilde{K} A z_{2}(X) \stackrel{\psi}{\longrightarrow} H^{1}\left(X ; Z_{2}\right) \times H^{2}\left(X ; Z_{2}\right) \longrightarrow 0 .
$$

Proof. Diagram (5) leads to the following diagram, after checking surjectivity and injectivity of a few more maps:

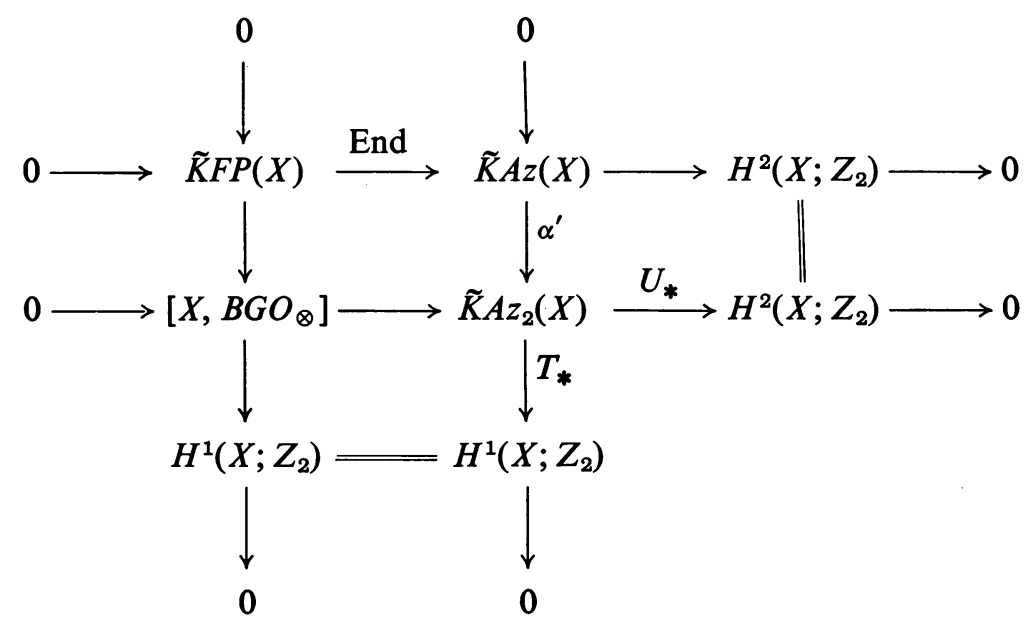

We extract from this a diagonal sequence

$$
\tilde{K} F P(X) \stackrel{\alpha^{\prime} \circ \text { End }}{\longrightarrow} \tilde{K} A z_{2}(X) \stackrel{T_{*} \times U_{*}}{\longrightarrow} H^{1}\left(X ; Z_{2}\right) \times H^{2}\left(X ; Z_{2}\right),
$$

which by definition and diagram chase yields the proposition.

There are two possible group structures for $H^{1} \times H^{2}$. This is seen by computing $[X \wedge X, X]$ for $X=K\left(Z_{2}, 1\right) \times K\left(Z_{2}, 2\right)$ [1]. We shall compute the maps $\mathrm{Cl}$ and $L$ of diagram (3) and in the process discover which group is present. The outline of this is as follows. 
By taking Grassmann manifolds as test spaces and using naturality we see that the map $L$ must be expressible in terms of Stiefel-Whitney classes. We will show by considering the Möbius bundle $M$ over $S^{1}$ that $w_{1}$ is present, and by considering the Hopf bundle $\eta$ over $S^{2}$ that $w_{2}$ is present. Then the map $L$ must be either $\left(w_{1}, w_{2}\right)$ or $\left(w_{1}, w_{1}^{2}+w_{2}\right)$. As neither of these is a homomorphism into $H^{1}\left(X ; Z_{2}\right)$ $\oplus H^{2}\left(X ; Z_{2}\right)$ we conclude that the group present is $1 \oplus H^{1}\left(X ; Z_{2}\right) \oplus H^{2}\left(X ; Z_{2}\right)$. We then just have to consider these two possibilities for the map.

We begin by examining $\mathrm{Cl}(1,1)$. Let $d_{1}$ and $d_{2}$ be a basis for $R^{1} \oplus R^{1}$ with the quadratic form $q\left(a d_{1}+b d_{2}\right)=-a^{2}+b^{2}$. Then $\mathrm{Cl}(1,1)$ has an algebra basis $d_{1}, d_{2}$ with $d_{1}^{2}=-1, d_{2}^{2}=+1$, and is isomorphic to $M_{1,1}$ if we use the correspondence

$$
d_{1} \rightarrow\left(\begin{array}{rr}
0 & 1 \\
-1 & 0
\end{array}\right), \quad d_{2} \rightarrow\left(\begin{array}{ll}
0 & 1 \\
1 & 0
\end{array}\right)
$$

Furthermore, $\mathrm{Cl}$ takes the four isometries of $O(1) \times O(1)$ of $R^{1} \oplus R^{1}$ to inner automorphisms of $\mathrm{Cl}(1,1)$ and of $M_{1,1}$ as follows:

$$
\begin{gathered}
\left(\begin{array}{ll}
1 & 0 \\
0 & 1
\end{array}\right) \rightarrow \pm 1 \rightarrow \pm\left(\begin{array}{ll}
1 & 0 \\
0 & 1
\end{array}\right) \\
\left(\begin{array}{rr}
-1 & 0 \\
0 & 1
\end{array}\right) \rightarrow \pm d_{2} \rightarrow \pm\left(\begin{array}{ll}
0 & 1 \\
1 & 0
\end{array}\right) \\
\left(\begin{array}{rr}
1 & 0 \\
0 & -1
\end{array}\right) \rightarrow \pm d_{1} \rightarrow \pm\left(\begin{array}{rr}
0 & 1 \\
-1 & 0
\end{array}\right) \\
\left(\begin{array}{rr}
-1 & 0 \\
0 & -1
\end{array}\right) \rightarrow \pm d_{1} d_{2} \rightarrow \pm\left(\begin{array}{rr}
1 & 0 \\
0 & -1
\end{array}\right) .
\end{gathered}
$$

Proposition 3.10. The map $L: \tilde{K} O\left(S^{1}\right) \rightarrow H^{1}\left(S^{1} ; Z_{2}\right)$ sends the generator $\{M\}$ of $\widetilde{K} O\left(S^{1}\right)$ to $w_{1}(M)$, the nonzero element of $H^{1}\left(S^{1} ; Z_{2}\right)$.

Proof. We consider the bundle $M \oplus \varepsilon$ over $S^{1}$, with $M$ having a negative definite and $\varepsilon$ a positive definite metric. Let $x$ and $y$ be the two points of $S^{0} \subset S^{1}$. Then the clutching function for $M \oplus \varepsilon$ sends $x$ to $\left(\begin{array}{ll}1 & 0 \\ 0 & 1\end{array}\right)$ in $O(1) \times O(1)$ and $y$ to $\left(\begin{array}{rr}-1 & 0 \\ 0 & 1\end{array}\right)$. Therefore the clutching function for $\mathrm{Cl}(M \oplus \varepsilon)$ sends $x$ to inner automorphism by $\left(\begin{array}{ll}1 & 0 \\ 0 & 1\end{array}\right)$ and $y$ to inner automorphism by $\left(\begin{array}{ll}0 & 1 \\ 1 & 0\end{array}\right)$. This graded algebra bundle defines the line bundle $M$ over $S^{1}$ again, as its clutching functions are $x \rightarrow+1$ and $y \rightarrow-1$ (cf. 3.8). By the usual correspondence between line bundles and $H^{1}\left(S^{1} ; Z_{2}\right)$ we get $w_{1}(M)$.

Next we discuss $\mathrm{Cl}(2,2)$. Suppose $d_{1}, d_{2}, d_{3}, d_{4}$ are the basis for $R^{2} \oplus R^{2}$ with the quadratic form $q\left(a d_{1}+b d_{2}+c d_{3}+d d_{4}\right)=-a^{2}-b^{2}+c^{2}+d^{2}$. Then $\mathrm{Cl}(2,2)$ has algebra basis elements $d_{1}, d_{2}, d_{3}, d_{4}$ with $d_{1}^{2}=d_{2}^{2}=-1$ and $d_{3}^{2}=d_{4}^{2}=+1$. To get matrix formulas we use the fact that $\mathrm{Cl}(2,2) \cong \mathrm{Cl}(1,1) \hat{\otimes} \mathrm{Cl}(1,1)$, so that generators of $\mathrm{Cl}(2,2)$ are given in terms of those of $\mathrm{Cl}(1,1)$ by $d_{1}=d_{1} \hat{\otimes} 1$, $d_{2}=1 \hat{\otimes} d_{1}, d_{3}=d_{2} \hat{\otimes} 1, d_{4}=1 \hat{\otimes} d_{2}$. Using this, the matrix interpretation of $\mathrm{Cl}(1,1)$, and Lemma 3.5, we get an isomorphism between $\mathrm{Cl}(2,2)$ and $M_{2,2}$ : 

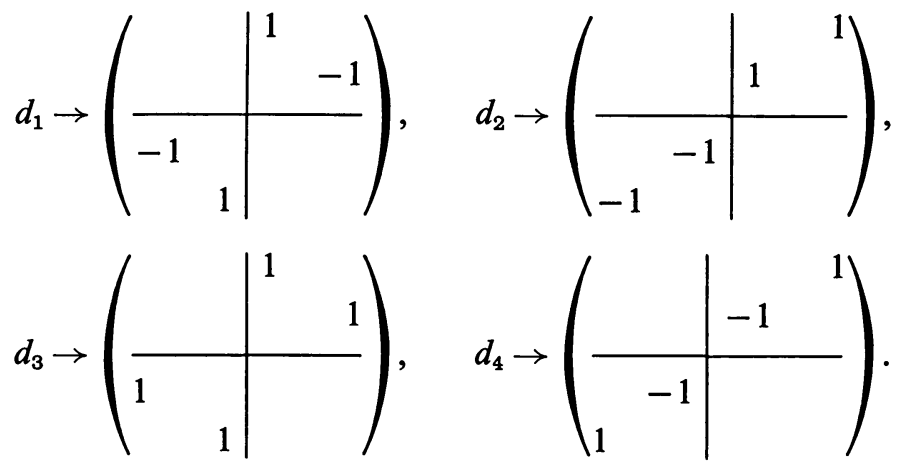

LEMMA 3.11. The fundamental group of $P O$ is $Z_{2}$ and of $P O(n)$ is given by

$$
\begin{aligned}
& \pi_{1} P O(n)=Z, \quad n=2 \text {, } \\
& =Z_{2}, \quad n \text { odd, } n>1 \text {, } \\
& =Z_{4}, \quad n \equiv 2(\bmod 4), \quad n>2 \text {, } \\
& =Z_{2} \oplus Z_{2}, \quad n \equiv 0(\bmod 4), \quad n>2 \text {. }
\end{aligned}
$$

Proof. The fibrations $Z_{2} \rightarrow O_{\otimes} \rightarrow P O$ and $Z_{2} \rightarrow O(n) \rightarrow P O(n)$ show all but, in the case $n$ even and $n>2$, which group of order 4 is present. In this case we can restrict to $S O(n)$ and $P S O(n)$. The simply connected covering space of $P S O(n)$ is Spin $(n)$, and the fiber over the identity is the subgroup $\{1,-1, \omega,-\omega\}$ where $\omega$ is the product of all the algebra generators of $\mathrm{Cl}(n, 0)$. As

$$
\begin{aligned}
\omega^{2} & =+1, & n \equiv 0 & (\bmod 4), \\
& =-1, & n \equiv 2 & (\bmod 4)
\end{aligned}
$$

we arrive at the group claimed.

Proposition 3.12. The map $L: \tilde{K} O\left(S^{2}\right) \rightarrow H^{2}\left(S^{2} ; Z_{2}\right)$ sends the generator $\{\eta\}$ of $\tilde{K} O\left(S^{2}\right)$ to $w_{2}(\eta)$, the nonzero element of $H^{2}\left(S^{2} ; Z_{2}\right)$.

Proof. We consider the bundle $\eta \oplus 2 \varepsilon$ over $S^{2}$, with $\eta$ having a negative definite and $2 \varepsilon$ a positive definite metric. Since by Proposition $3.9 \widetilde{K} A z_{2}\left(S^{2}\right) \cong G B^{\sim}\left(S^{2}\right) \cong Z_{2}$, and $\widetilde{K} A z_{2}\left(S^{2}\right) \cong \pi_{1}(P G O)$, it suffices to show that the clutching function of $\mathrm{Cl}(\eta \oplus 2 \varepsilon)$ represents the nonzero element of $\pi_{1}(P G O)$.

The clutching function $g: S^{1} \rightarrow S O(2)$ for $\eta$ is given by

$$
g(\theta)=\left(\begin{array}{rr}
\cos \theta & -\sin \theta \\
\sin \theta & \cos \theta
\end{array}\right), \quad 0 \leqq \theta<2 \pi .
$$

Hence that of $\eta \oplus 2 \varepsilon$ is

$$
\left(\stackrel{g(\theta)}{ } \mid \frac{}{I_{2}}\right)
$$


in $O(2) \times O(2)$. By $\mathrm{Cl}$ these isometries of $R^{2} \oplus R^{2}$ give rise to automorphisms of $\mathrm{Cl}(2,2)$. By direct computation on basis elements one sees that $\mathrm{Cl}$ must take

$$
\left(\begin{array}{l|l}
g(\theta) & \\
I_{2}
\end{array}\right)
$$

to inner automorphism of $\mathrm{Cl}(2,2)$ by $\cos \theta / 2+\sin (\theta / 2) d_{1} d_{2}$, thus to inner automorphism of $M_{2,2}$ by

$$
\left(\frac{g(\theta / 2)}{g(\theta / 2)}\right) .
$$

Let $h(\theta)=g(\theta / 2)$. Clearly the generator of $\pi_{1} P S O(2)$ which is not hit by the projection from $\pi_{1} S O(2)$ is $[h]$.

Consider the following diagram:

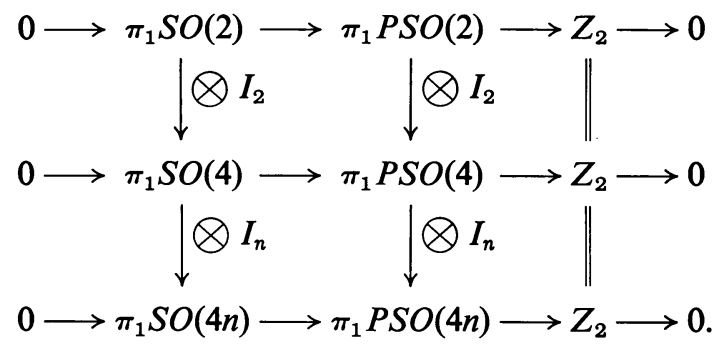

The groups in the left column are all $Z_{2}$ except $\pi_{1} S O(2)=Z$, while the center column is given by Lemma 3.11. In the left column, $\otimes I_{n}$ is zero for $n$ even, an isomorphism for $n$ odd. In the center column it follows that [h] goes nontrivially to $\pi_{1} P S O(4)$ and in fact goes to the generator of $\pi_{1} P O$ in the limit. As $\Delta^{\prime}(h(\theta))$ is the clutching function for $\mathrm{Cl}(\eta \oplus 2 \varepsilon)$, it follows since $\alpha^{\prime}$ is a monomorphism that $\{\mathrm{Cl}(\eta \oplus 2 \varepsilon)\}$ is nonzero.

Proposition 3.13. The maps $\mathrm{Cl}$ and $L$ are given by

$$
\mathrm{Cl}(\{E\},\{F\})=\left(1+w_{1}(E)+w_{2}(E)\right)\left(1+w_{1}(F)+w_{1}(F)^{2}+w_{2}(F)\right)
$$

and $L(\{E\})=1+w_{1}(E)+w_{2}(E)$.

Proof. We use the canonical line bundle $\zeta$ over the real projective plane. As in Proposition 3.10, the coordinate transformations for $\mathrm{Cl}(\zeta \oplus \varepsilon)$ take values either $\pm\left(\begin{array}{ll}1 & 0 \\ 0 & 1\end{array}\right)$ or $\pm\left(\begin{array}{ll}0 & 1 \\ 1 & 0\end{array}\right)$ in $P G O(1)$. If we construct a bundle using in corresponding places the coordinate transformations $+\left(\begin{array}{ll}1 & 0 \\ 0 & 1\end{array}\right)$ and $+\left(\begin{array}{ll}0 & 1 \\ 1 & 0\end{array}\right)$ we get a bundle in $[X, B G O(1)]$ which maps to $\mathrm{Cl}(\zeta \oplus \varepsilon)$, showing that $\mathrm{Cl}(\zeta \oplus \varepsilon)$ must map to zero in $H^{2}\left(R P^{2} ; Z_{2}\right)$. We conclude that $\mathrm{Cl}(\zeta \oplus \varepsilon)$ maps to $1+w_{1}(\zeta)$ in $G B^{\sim}\left(R P^{2}\right)$. As $\mathrm{Cl}(\zeta \oplus \zeta)$ must map to 1 in $G B^{\sim}\left(R P^{2}\right)$, it follows that $\mathrm{Cl}(\varepsilon \oplus \zeta)$ maps to $1+w_{1}(\zeta)+w_{1}(\zeta)^{2}$. This completes the proof of Theorem 3.1.

Parallel to Proposition 2.5 we have 
COROLlARY 3.14. The following diagram is exact and commutative.

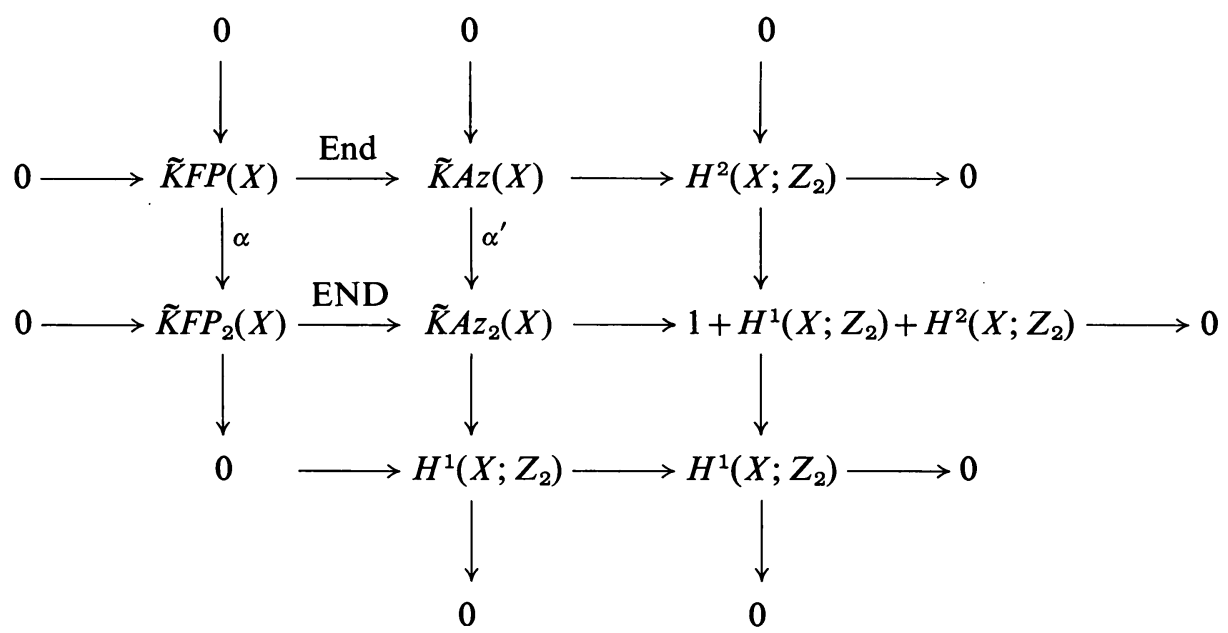

Finally, using $X=R P^{2}$ one sees that the vertical sequences need not split.

\section{BIBLIOGRAPHY}

1. M. Arkowitz and C. R. Curjel, On the number of multiplications of an H-space, Topology 2 (1963), 205-209. MR 27 \#2985.

2. M. F. Atiyah, R. Bott and A. Shapiro, Clifford modules, Topology 3 (1964), suppl. 1, 3-38. MR 29 \#5250.

3. H. Bass, Topics in algebraic K-theory, Mimeographed Notes, Tata Institute, Bombay, India, 1967.

4. A. Delzant, Définition des classes de Stiefel-Whitney d'un module quadratique sur un corps de caractéristique différente de 2, C. R. Acad. Sci. Paris 255 (1962), 1366-1368. MR 26 \#175.

5. A. Dold, Halbexakte Homotopiefunktoren, Lecture Notes in Math., No. 12, SpringerVerlag, Berlin, 1966.

6. P. Donovan and M. Karoubi, Graded Brauer groups and K-theory with local coefficients, (to appear).

7. - Groupe de Brauer et coefficients locaux en K-théorie, C. R. Acad. Sci. Paris 269 (1969), 387-389.

8. A. Grothendieck, Algèbres d'Azumaya et interprétations diverse, Séminaire Bourbaki, 17e année (1964/65), exposé 290, reprint, Benjamin, New York, 1964/65. MR 33 \#5420L.

9. S. Helgason, Differential geometry and symmetric spaces, Pure and Appl. Math., vol. 12, Academic Press, New York, 1962. MR 26 \#2986.

10. W. C. Hsiang, A note on free differentiable actions of $S^{1}$ and $S^{3}$ on homotopy spheres, Ann. of Math. (2) 83 (1966), 266-272. MR 33 \#731.

11. J. Milnor, Construction of universal bundles. II, Ann. of Math. (2) 63 (1956), 430-436. MR 17, 1120.

12. - Microbundles. I, Topology 3 (1964), suppl. 1, 53-80. MR 28 \#4553b.

13. W. Scharlau, Quadratische Formen und Galois-Cohomologie, Invent. Math. 4 (1967), 238-264. MR 37 \#1442. 
14. M. Spivak, Spaces satisfying Poincaré duality, Topology 6 (1967), 77-101. MR 35 \#4923.

15. N. Steenrod, The topology of fibre bundles, Princeton Math. Series, vol. 14, Princeton Univ. Press, Princeton, N. J., 1951. MR 12, 522.

16. C. T. C. Wall, Graded Brauer groups, J. Reine Angew. Math. 213 (1963/64), 187-199. MR $29 \# 4771$.

\section{NORTHWESTERN UNIVERSITY,}

EVANSTON, ILLINOIS 60201 